\title{
RADIO OBSERVATIONS OF THE SOLAR GORONA AT SUNSPOT MINIMUM
}

\section{A. HEWISH}

Cavendish Laboratory, Cambridge, England

Previous experiments to determine the distribution of radio brightness across the solar disk at metre wave-lengths using the method of Fourier synthesis have been described by O'Brien (1953) [1]. These results could be explained in terms of a spherically symmetrical corona only if the

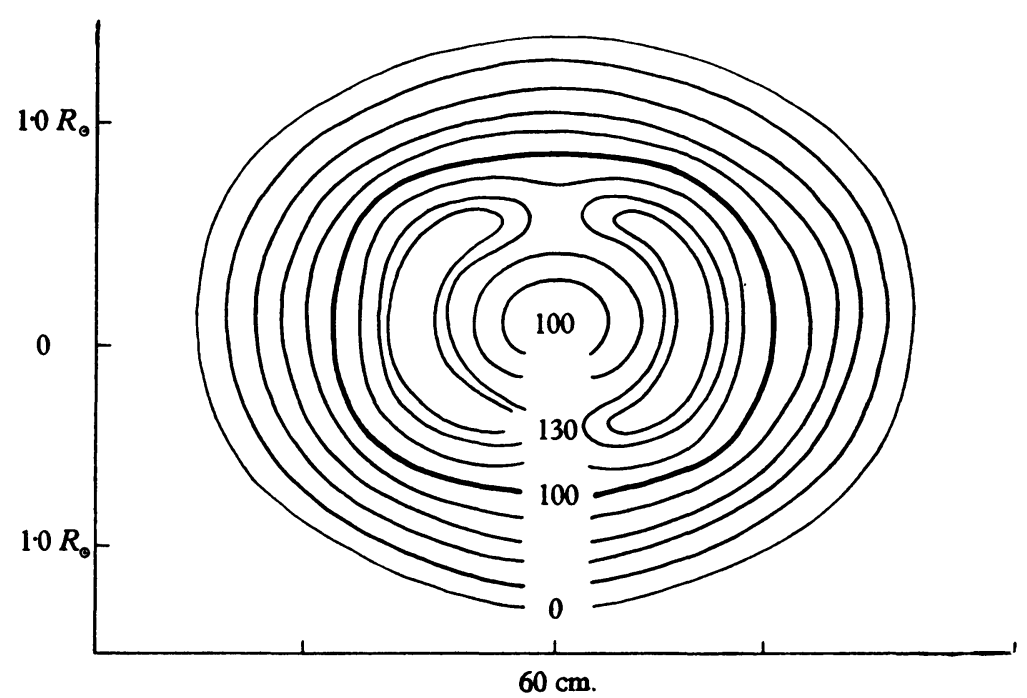

Fig. I. The distribution of brightness across the solar disk at $60 \mathrm{~cm}$.

electron density and temperature differed appreciably from estimates derived by visual methods (O'Brien and Bell, 1954) [2]. In order to check these measurements a similar series of observations was carried out in 1954 during an extended period of exceptionally low solar activity. Additional experiments, which gave information concerning the ellipticity of the brightness distribution, were made at Cambridge during the partial eclipse of June I954. 
Interferometers of variable spacing in an east-west direction were used on wave-lengths of $\mathrm{r} \cdot 4,3.7$ and 7.9 metres; on a wave-length of $60 \mathrm{~cm}$. more detailed observations were made in which the direction of the interferometer axis was varied over a wide range of angles. The $60 \mathrm{~cm}$. experiments enabled the complete distribution to be derived assuming symmetry about the rotation axis only, and this assumption was justified by the consistency of the observations over a period of several weeks. The results are shown in Fig. I where it is seen that the contours are considerably flattened towards the poles.

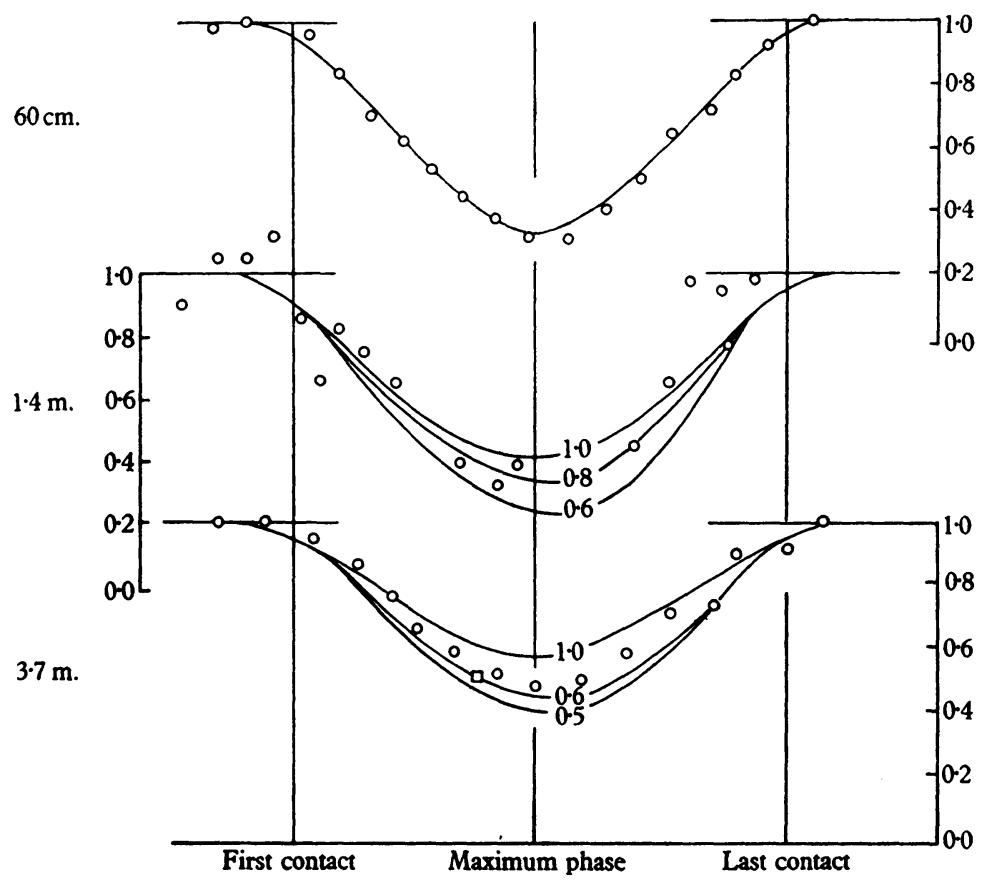

Fig. 2. Observations obtained during the partial eclipse of 1954 at Cambridge. At $1 \cdot 4$ and 3.7 metres the smooth curves denote the results derived theoretically for models of different axial ratio. At $60 \mathrm{~cm}$. the smooth curve is that derived from the distribution shown in Fig. 1 .

At the metre wave-lengths it was not possible to derive the complete distribution unambiguously since the direction of the interferometer was fixed. The method adopted was to assume that the contours were elliptical, the degree of ellipticity being derived independently from eclipse measurements.

The eclipse measurements are shown in Fig. 2. At a wave-length of $60 \mathrm{~cm}$. the shape of the eclipse curve was in excellent agreement with that 
expected from the distribution obtained by the interferometer method. At metre wave-lengths the eclipse results indicated an appreciable degree of ellipticity, and the best fit was obtained for models having an axial ratio of 0.8 at $\mathrm{r} \cdot 4$ metres and 0.6 at 3.7 metres.

Using these results the distributions shown in Fig. 3 were obtained. The curves are largely similar to those reported previously by O'Brien, but the
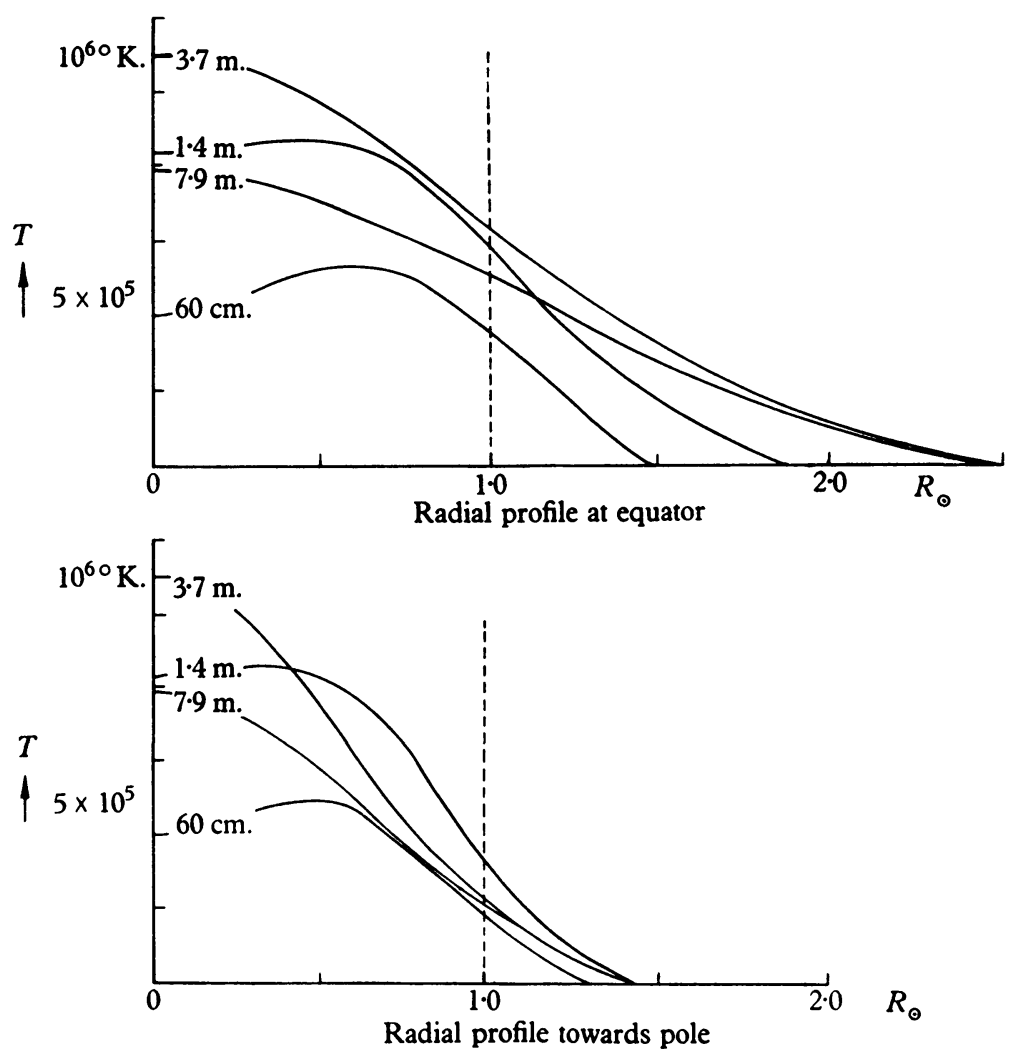

Fig. 3. Radial profiles of the brightness distribution taken along the polar axis and in the equatorial plane.

absolute brightness has been modified on account of the ellipticity of the contours. The radio model of the corona derived by O'Brien and Bell on the basis of spherical symmetry must similarly be modified, and it now seems probable that the radio observations may be explained without the necessity for a rapidly falling temperature in the outer corona. In addition, limb brightening has now been detected at $60 \mathrm{~cm}$. The absence of limb 
brightening in the earlier measurements of Stanier (1950) [3] could be due to the presence of long-lived regions of enhanced emission which might be a permanent feature on the disk at times other than sunspot minimum.

\section{REFERENCES}

[1] O'Brien, P. A. M.N.R.A.S. 113, 597, 1953.

[2] O'Brien, P. A. and Bell, C. J. Nature, 173, 219 , I954.

[3] Stanier, H. M. Nature, 165, 354, 1950. 